\title{
HCI Summer School in Tallinn
}

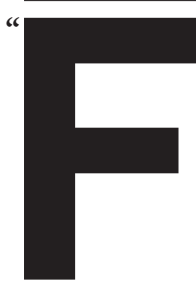

un and foundational. It was a great experience. Made a lot of good friends. All the program committee members were very, very helpful."

"We learned a lot about research in HCI. Very good university facilities."

"Good selection of topics."

"Very well-organized team, friendly, helpful, and supportive in every possible way. I learned a lot. Also about Estonian history, culture, and traditions through the interesting talks during social activities."

"Good teachers, useful material."

These were some of the experiences of students who took part in the first summer school on "Research Methods in Human-Computer Interaction (HCI)" in Tallinn, Estonia from July 25-31, 2014. SIGCHI sponsored the event with 25 scholarships for HCI students from all over Europe.

Co-organized by SIGCHI local chapters EstCHI and Cyprus SIGCHI and hosted by Tallinn University's Institute of Informatics, the first summer school on research methods in HCI was a big success. It was led by David Lamas, head of the Interaction Design Lab at the Institute of Informatics, Tallinn University; Panayiotis Zaphiris, dean of the School of Fine and Applied Arts and director of the Cyprus Interaction Lab at Cyprus University of Technology; and
Effie Lai-Chong Law from the University of Leicester, U.K.

Attended by 38 Ph.D. students and practitioners specializing in HCI from more than 20 different countries, it gave a chance to all attendees to take part in training, discussions, networking, and social activities.

The course was designed primarily to help Ph.D. students and practitioners better understand the major research methods in HCI and allow them to combine and use these methods to tackle their research objectives.

The course included lectures from prominent academics and practitioners in the area of HCI. Duncan Brumby from University College London addressed Experimental Design; Jettie Hoonhout from Philips Research looked into Ethics, Questionnaire and Survey Design; Geraldine Fitzpatrick from TU Wien, Austria, introduced and explored Ethnographic Methods for User Research; Effie Law covered User Experience Evaluation Methods; Katrin Niglas from Tallinn University provided an introduction to designing research with Mixed Methods; and finally, Sian Lindley from Microsoft Research addressed Narrative-Based Methods.

Throughout the course, students worked in groups exploring ideas and proposing solutions to real research problems. Overall, the environment was positive and the feedback was encouraging. Students found the course valuable, the teachers insightful, and the experience well-organized and welcoming.

Further, the course was run as part of the annual Tallinn Summer School. Thus, the learning experience was enriched with revelations "about Estonian history, culture, and traditions through interesting talks during social activities."

The course was credited with 4 ECTS (European Credit Transfer and Accumulation System) credit points by Tallinn University through their Open University department. It was made possible through the generous support of ACM SIGCHI, the Estonian Information Technology Foundation for Education, and Tallinn University.

This year's course takes place July 6-10, 2015 in Limassol, Cyprus, and will be hosted by the Cyprus University of Technology.

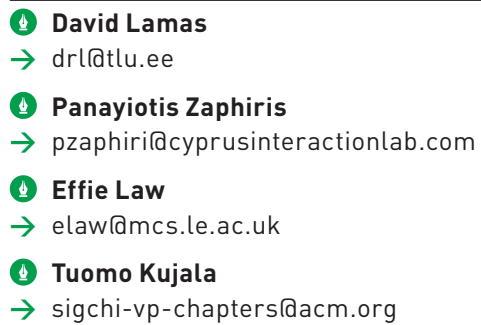

(a) http://idlab.tlu.ee/rmhci/

() https://www.facebook.com/ events/702865866421779 